\title{
Intermédialités
}

Histoire et théorie des arts, des lettres et des techniques

Intermediality

History and Theory of the Arts, Literature and Technologies

\section{Introduction. Le reenactment ou le répertoire en régime intermédial}

\section{Anne Bénichou}

Numéro 28-29, automne 2016, printemps 2017

refaire

redoing

URI : https://id.erudit.org/iderudit/1041075ar

DOI : https://doi.org/10.7202/1041075ar

Aller au sommaire du numéro

Éditeur(s)

Revue intermédialités (Presses de l’Université de Montréal)

ISSN

1920-3136 (numérique)

Découvrir la revue

Citer ce document

Bénichou, A. (2016). Introduction. Le reenactment ou le répertoire en régime

intermédial. Intermédialités / Intermediality, (28-29).

https://doi.org/10.7202/1041075ar 


\title{
Introduction. Le reenactment ou le répertoire en régime intermédial
}

\author{
ANNE BÉNICHOU
}

epuis le début de ce millénaire, on observe dans les pratiques artistiques et culturelles, et dans les discours spécialisés, un certain engouement pour le reenactment. Ce terme qui n'a pas d'équivalent satisfaisant en français désigne les phénomènes de recréation, de reconstitution, de reprise et d'autres formes de réactivation vivante d'œuvres performatives du passé, d'événements historiques ou de phénomènes culturels. Il se compose du verbe « enact », qui signifie « jouer » au sens théâtral ou « promulguer » au sens juridique, et du préfixe « re- », qui indique aussi bien « le retour à un état antérieur », « un changement de direction », « un renforcement » ou « la répétition d'une action ${ }^{\mathrm{I}} \gg$. L'Oxford Dictionary en donne la définition suivante : «The acting out of a past event », illustrée d'exemples tels la recréation d'une bataille du $19^{\mathrm{e}}$ siècle, l'écriture ( $\ll$ Writing is frequently a form of reenactment »), la téléréalité2, insistant sur la multiplicité des formes médiales de la remise en acte. Quant au Cambridge Dictionary, il évoque d'abord une fidélité à l'événement passé et à l'original : « If you re-enact an event, you try to make it happen again in exactly the same way it happened the first time $[\ldots]^{3} \gg$. Puis, il nuance en situant le reenactment dans les domaines du divertissement ou du souvenir : « [...] often as an entertainment or as a way to help people remember certain facts about an event $[\ldots]^{4} \gg$. Pour pallier les difficultés de la traduction du terme en français, Aline Caillet propose « reconstitution jouées ». L'expression reconduit le paradoxe entre le désir d'un retour à un état initial (la reconstitution) et son dépassement à travers le jeu et l'interprétation.

\footnotetext{
I Office québécois de la langue française, «Re- », http://bdl.oqlf.gouv.qc.ca/bdl /gabarit_bdl.asp?id=1677 (consultation le ro juillet 2017).

${ }^{2}$ Oxford Dictionary, « re-enactment », https://en.oxforddictionaries.com/definition/reenactment (consultation le ro juillet 2017).

3 Cambridge Dictionary, « re-enact», http://dictionary.cambridge.org/dictionary /english/re-enact (consultation le ro juillet 2017).

4 Ibid.

5 Aline Caillet, «Le re-enactment: refaire, rejouer ou répéter l'histoire ? », Marges, ${ }^{\circ}{ }_{17}$, 2013, p. 67.
} 
Malgré ce flottement sémantique, le terme reenactment est de plus en plus utilisé et connaît même une fortune critique dans la littérature spécialisée en anglais comme en français. Dans les arts vivants, alors que les phénomènes de reprise sont inscrits dans une tradition et qu'une terminologie existe pour les différencier, on tend à les dénommer indistinctement « reenactment ». En danse, dans les années 1980, il est d'usage de distinguer les « reconstructions », les reprises et les recréations. Les premières concernent des œuvres chorégraphiques du passé qui n'ont pas été interprétées depuis longtemps et nécessitent pour les remonter des recherches dans les archives et le recours à d'anciens interprètes. Les deuxièmes désignent des œuvres que l'on n'a pas cessé de jouer et pour lesquelles on possède les connaissances et les savoir-faire. Les troisièmes autorisent une très grande liberté à l'égard de l'œuvre originale $^{6}$. Si ces distinctions ne sont pas obsolètes, depuis les années 2000, le terme reenactment tend à les englober, comme le fait remarquer André Lepecki ${ }^{7}$ Cette mutation indique que la référence à l'œuvre d'origine n'est plus pensée en termes de fidélité, mais selon d'autres enjeux.

I3 Lorsque les universitaires se sont intéressés au reenactment à la fin du $20^{\mathrm{e}}$ siècle, ils n’ont pas cherché à en restreindre le champ sémantique, mais l'ont au contraire étendu à d'autres domaines, notamment les nouveaux médias. Le mot désigne désormais des phénomènes issus du théâtre, de la danse, des arts visuels, de la littérature, de l'histoire vivante, de l'historiographie, du cinéma, de la télévision, des expositions muséales, des récits de voyage, des jeux vidéo, des mondes virtuels, etc. Cette diversité oblige à adopter des approches interdisciplinaires, intermédiales, et à aménager des passerelles entre les pratiques culturelles dites savantes et populaires, professionnelles et amateurs, artistiques et non artistiques.

Le succès du terme reenactment, son élargissement sémantique et le nivellement de pratiques très différentes qu'il opère évoquent la fortune critique que connut le terme « archive » au cours des années 1990. Comme lui, il cherche moins à circonscrire avec précision des pratiques, mais indique plutôt l'émergence de nouvelles sensibilités historiques, de relations autres avec le passé. En quoi consistent-

\footnotetext{
${ }^{6}$ Alice Carré, Marion Rhéty et Ariane Zaytzeff, « Le fantôme, le monument et le combustible », Agôn, $\mathrm{n}^{\circ} 6$ 《La Reprise », 20I4, http://agon.ens-lyon.fr/index.php?id=2806 (consultation le ro juillet 2017).

7 André Lepecki, « Le corps comme archive. Volonté de réinterpréter et survivances de la danse », traduction de Aude Tincelin, dans Anne Bénichou (dir.), Recréer/Scripter. Mémoires et transmissions des auvres performatives et chorégraphiques contemporaines, Dijon, Les presses du réel, 2015, p. 33-70.
} 
elles ? L'engouement pour le reenactment manifeste un désir de réintégrer le corps, les affects, le performatif dans notre rapport au passé et dans les représentations que nous en proposons. Parce qu'il met en dialogue des temps hétérogènes, le passé et le présent, souvent même des historicités plurielles, le reenactment joue de l'anachronisme et de l'inactuel, au sens où l'entend Giorgio Agamben. Il exploite leur capacité à mieux nous faire voir le temps présent et à « lire l'histoire de manière inédite, [à] la citer en fonction d'une nécessité ${ }^{8} \gg$. Son succès dans les sphères savantes et amateurs est le signe d'une certaine démocratisation de l'histoire qui n'est désormais plus l'apanage des spécialistes. L'histoire reenactée est une histoire négociée entre divers acteurs. Enfin, le reenactment relance les débats sur les rapports entre l'archive et la mémoire, et permet de les repenser non pas en termes d'exclusions (le triomphe moderne de l'archive sur la mémoire ou son corollaire postmoderne, le règne de l'archive ébranlé par les mémoires), mais d'intrication dialectique permettant de déverrouiller les consensus, de relancer les débats.

Cette relation et cette sensibilité autres au passé semblent procéder de la convergence de trois phénomènes historiques, théoriques et artistiques que j'aborderai successivement dans cet essai introductif. La tradition des pageants historiques qui remonte à la fin ${\mathrm{du} \mathrm{I}^{\mathrm{e}}}^{\mathrm{e}}$ siècle se réactualise à travers les reenactments, les musées d'histoire vivante et, plus récemment, les univers médiatiques et technologiques. Les débats théoriques issus des performance studies réhabilitent le performatif, les contre-mémoires et les savoirs des corps, tandis qu'une nouvelle génération d'historiens croise l'historiographie et les études culturelles pour comprendre le potentiel émancipateur du reenactment dans les fabriques de l'histoire. Enfin, les pratiques artistiques s'emparent de la culture du reenactment, de ses objets, procédés et tactiques, pour les importer dans les cultures de l'image, selon des processus de négociation que j’appelle « le répertoire en régime intermédial ».

\section{DES PAGEANTS À SECOND LIFE :}

\section{LE JEU DE L'IMMERSION ET DE LA DISTANCIATION}

Bien que les discours savants sur le reenactment et son appropriation par les pratiques artistiques soient assez récents, le phénomène s'inscrit dans une longue tradition restée peu étudiée. Sa pratique remonte à la fin du $19^{\mathrm{e}}$ siècle, en Angleterre, aux États-Unis, au Canada et en Australie surtout, sous la forme de pageants

\footnotetext{
${ }^{8}$ Giorgio Agamben, Qu'est-ce que le contemporain?, traduction de Maxime Rovere, Paris, Éditions Payot \& Rivages, 2008, p. 39.
} 
historiques modernes qui poursuivent eux-mêmes la tradition des pageants religieux issus du Moyen Âge, encore appelés jeux, miracles et mystères ${ }^{9}$. Ces spectacles à grand déploiement, généralement en plein air, pouvant accueillir des milliers de spectateurs, relataient des épisodes de l'histoire de la ville ou de la localité où ils se tenaient. Ils célébraient l'anniversaire d'un événement, la fondation d'une ville ou d'une institution. Certains pageants privilégiaient les faits historiques, alors que d'autres prenaient plus de liberté à l'égard de l'histoire et jouaient sur le registre allégorique, illustrant des valeurs morales, patriotiques, nationales, etc. Commandés par les pouvoirs politiques et les élites sociales, ces spectacles représentaient leur conception de l'histoire. La distribution des rôles parmi les membres de la communauté reflétait souvent les hiérarchies socio-économiques et leur reproduction à travers les générations. Les pageants constituaient un puissant outil de confirmation des valeurs et des normes sociales en favorisant l'adhésion et l'incorporation à travers la participation. Cet usage idéologique traverse toute l'histoire des reenactments. Que l'on pense aux actions de masse de la jeune URSS, au cours desquelles des épisodes de la Révolution de 1917 étaient rejoués en plein air, sur les lieux mêmes, par des nonprofessionnels ${ }^{\mathrm{IO}}$. Aujourd'hui, en Chine, dans le cadre du plan de développement $\mathrm{du}$ «tourisme rouge », le gouvernement encourage les reconstitutions des batailles de l'Armée populaire de libération lors de la Longue Marche ${ }^{\text {II }}$. Le public est invité à y prendre part.

Au début des années 1960 aux États-Unis, lors des festivités du centenaire de la guerre de Sécession, plusieurs grandes batailles historiques furent reconstituées sur leur site originel, amorçant une tradition qui n’a cessé depuis de se déployer. En Europe, on se mit également à reconstituer les batailles de l'ère napoléonienne (surtout la dernière période du règne de Napoléon $\mathrm{I}^{\mathrm{er}}$, appelée les Cent-Jours, se

9 Sur l'histoire du reenactment, voir entre autres : Stephen Gapps, Performing the Past: $A$ Cultural History of Historical Reenactments, thèse de doctorat, Sydney, University of technology, 2002; Sven Lütticken, «An Arena in Which to Reenact», dans Sven Lütticken (dir.), Life, Once More: Forms of Reenactment in Contemporary Art, catalogue d'exposition, Rotterdam, Witte de With, Center for Contemporary Art, 2005, p. 17-6o; Robert Blackson, «Once More... With Feeling: Reenactment in Contemporary Art and Culture », Art Journal, vol. 66, n I, mars 2007, p. 28-40; Inke Arns, « History Will Repeat Itself », dans Inke Arns et Gabriele Horn (dir.), History Will Repeat Itself. Strategies of Reenactment in Contemporary (Media) Art and Performance, catalogue d'exposition, Berlin, KW Institute for Contemporary Art, Francfort, Revolver, 2007, p. 38-63.

Io La plus célèbre, L'année rouge, commémorait le deuxième anniversaire de la révolution sur la place du Palais à Petrograd.

"I Anne Jaurès, « Le tourisme rouge en Chine : véritable engouement ou carte forcée ? », Monde chinois, vol. 29, $\mathrm{n}^{\circ}$ I, 20I2, p. 88-IOI. 
terminant par la défaite de ses troupes à Waterloo). Les événements dignes d'être recréés sont ceux qui figurent dans les livres d'histoire, la grande Histoire, les moments fondateurs d'une nation. Une véritable économie se mit en place: le tourisme de guerre et commémoratif, le marché des costumes d'époque et des accessoires. Les associations d'amateurs ne cessent depuis de se multiplier; leurs sites envahissent Internet, et YouTube regorge de captations des reenactments de grandes batailles.

Dans les années 1970, le démantèlement des empires coloniaux et l'affirmation des identités locales provoquent une diversification des événements historiques jugés dignes d'être rejoués. Les communautés réclament leurs propres histoires et la fin des métarécits porteurs des valeurs coloniales. Cette liberté de rejouer divers passés entraîne débats et controverses quant aux histoires « reenactables » ou non (comme on dit de certains événements qu'ils sont irreprésentables), quant à la distribution des rôles qui ne répond plus à la logique filiale des pageants ni à la communion patriotique des reenactments à saveur allégorique, quant aux groupes et aux communautés exclus des récits historiques. Cette redistribution des pouvoirs de la représentation historique exacerbe les sensibilités.

Un autre espace culturel où se pratiquent des formes de reenactment est le musée d'histoire vivante ${ }^{\mathrm{I} 2}$. Dans ces sites restaurés ou reconstitués, le personnel en costume vaque aux activités quotidiennes de l'époque. Les premiers furent créés à la fin des années 1920 aux États-Unis. Le Greenfield Village fondé en 1929 par Henry Ford dans la banlieue de Détroit (Michigan) retrace les moments importants des révolutions industrielles, du $\mathrm{I}^{\mathrm{e}}$ au $2 \mathrm{O}^{\mathrm{e}}$ siècle. En Virginie, le Colonial Williamsburg est une restauration de l'ancienne capitale de la colonie britannique de Virginie au I $8^{\mathrm{e}}$ siècle. Dans la lignée des pageants allégoriques, ce musée d'histoire vivante centre son propos sur les valeurs civiques de liberté et d'égalité, à l'origine même de « l'idée d'Amérique ${ }^{\mathrm{I} 3} \gg$.

\footnotetext{
${ }^{12}$ Sur les musées d'histoire vivante, voir entre autres: Richard Schechner, Performance. Expérimentation et théorie du théâtre aux USA, Anne Cuisset et Marie Pecorari(éd.), traduction de Marie Percorari et Marc Boucher, Montreuil-sous-Bois, Éditions théâtrales, 2008; Gapps, 2002; Scott Magelssen, Living History Museums. Undoing History Through Performance, Lanham (Maryland), Scarecrow Press, 2007.

13 Site Internet du Colonial Williamsburg: https://www.colonialwilliamsburg.com/ (consultation le ro juillet 2017).
} 


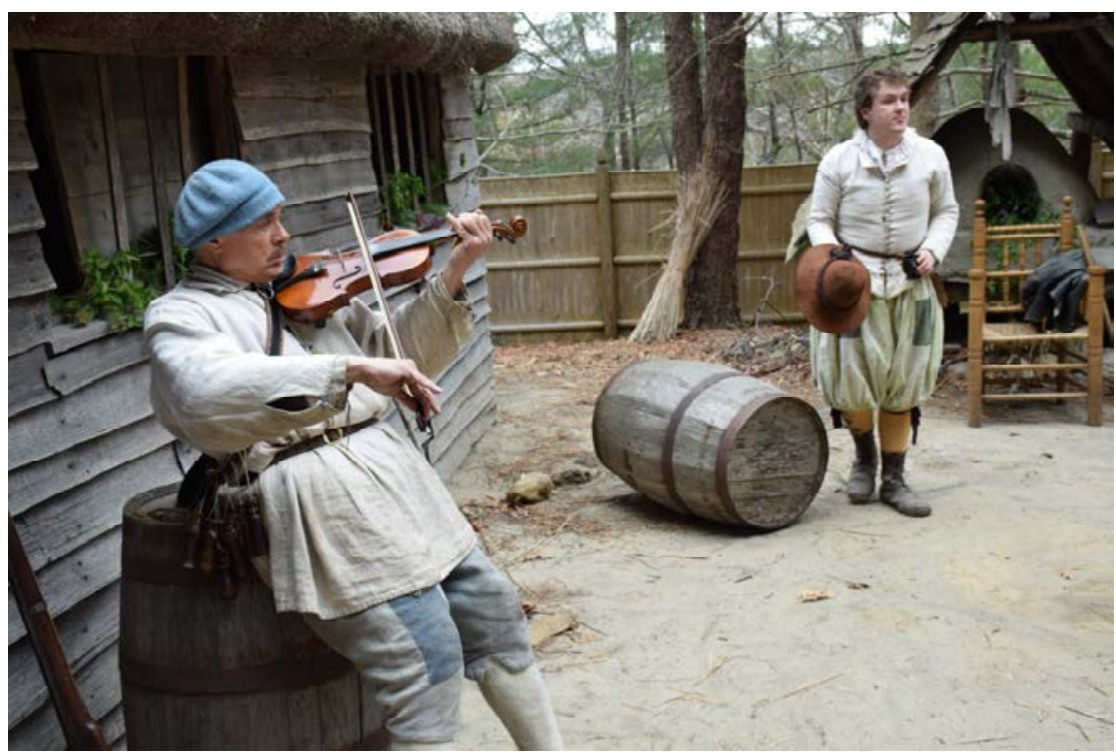

Fig. I. Todd van Hoosear, Plimoth Plantation, Plymouth, MA. Avec Michele Martin, photographie, ${ }^{\text {er }}$ mai 20I6. @ Todd Van Hoosear, 2016.

Certaines de ces institutions ont adopté la technique dite «à la première personne » selon laquelle les guides costumés incarnent des personnages historiques discourant au « je ». Cette approche est élaborée en 1978 à la Plimoth Plantation dans le Massachusetts, une recréation de la colonie de Plymouth du $17^{\mathrm{e}}$ siècle (voir la figure I). Les employés, tels des acteurs, jouent des habitants y ayant réellement vécu. Chacun reçoit une fiche manuscrite dans un anglais $d u{ }^{\mathrm{e}}$ siècle lui donnant des informations biographiques sur son personnage, ainsi qu'un enregistrement $\mathrm{du}$ dialecte de l'époque. Des recherches historiques, linguistiques et biographiques sont menées continuellement pour compléter et actualiser les données. Les interprètes comblent les lacunes en improvisant et en puisant dans leur propre vie, en interaction avec les visiteurs ${ }^{14}$.

Pageants, reenactments et histoire vivante procèdent d'une dialectique de l'immersion et de la distanciation, la première reposant sur une quête d'authenticité et des processus d'identification, la deuxième, sur l'anachronisme et la feintise. Le recours au langage à double sens et au simulacre permet d'activer ce dispositif dialectique complexe. La valeur d'authenticité est en effet centrale dans les

14 Richard Schechner, «La restauration du comportement», dans Performance. Expérimentation et théorie du théâtre aux USA, 2008, p. 397-464. 
reenactments. Elle n'est pas entendue au sens traditionnel de conformité à un original ou à un modèle. Elle désigne plutôt l'efficacité de la simulation, la capacité de s'absorber dans le jeu et de s'identifier à son personnage. Les reenactors ont développé une subtile gradation des degrés d'authenticitérs. Au bas de l'échelle, les farbs (far be it for me to tell them what they are doing wrong) performent dans des vêtements achetés au supermarché ou n'évoquant que vaguement la période historique rejouée. $\mathrm{Au}$ sommet, les authentic hardcores et superhardcores confectionnent leurs habits et accessoires au prix de minutieuses recherches ${ }^{16}$. Ils tentent d'éprouver « une expérience physique de l'authenticitér7 ${ }^{\gg}$ en se surpassant physiquement dans le but d'atteindre un état d'immersion ou d'absorption complète dans l'événement «reenacté ». Tony Horwitz qualifie ces moments d'acmé de «period rush», «a momentary illusion of actually being in the past ${ }^{18} \gg$.

Paradoxalement, les temporalités les plus hétérogènes cohabitent dans le reenactment. On y est simultanément dans le présent et dans le passé, et souvent dans plusieurs passés à la fois. Au Greenfield Village, on croise aussi bien une Ford $\mathrm{T} \mathrm{du}$ début $\mathrm{du} 2 \mathrm{O}^{\mathrm{e}}$ siècle qu'une charrette. Des personnages du $\mathrm{I}^{\mathrm{e}}$ et du $2 \mathrm{O}^{\mathrm{e}}$ siècle conversent avec des touristes du $2 \mathrm{I}^{\mathrm{e}}$ siècle. À la Plimoth Plantation, bien que le site ait été recréé à la fin des années 1940 , on y parle un anglais du $17^{\mathrm{e}}$ siècle. Dans les reenactments des batailles de la guerre de Sécession, les spectateurs avec leurs chaises pliantes et leurs appareils électroniques côtoient les soldats nordistes et sudistes en uniforme.

Comment concilier ces anachronismes éhontés avec la quête d'authenticité et d'immersion ? La ruse consiste à « faire comme si » le temps du jeu. La notion de « feintise ludique » de Jean-Marie Schaeffer est ici fort utile ${ }^{19}$. Parfaitement résumée dans l'expression «on le fait pour de faux », que les enfants affectionnent, elle consiste à produire des leurres permettant l'immersion dans des univers de fiction : $\ll[\ldots]$ il ne s'agit pas d'induire en erreur, mais de mettre à la disposition de celui qui

is Stephen Gapps, «Mobile Monuments: A View of Historical Reenactment and Authenticity from Inside the Costume Cupboard of History », Rethinking History, vol. I3, $\mathrm{n}^{\mathrm{o}}$ 3, septembre 2009, p. 395-409.

${ }^{16}$ Entre les deux : le mainstreamer, le faux progressive, le progressive, l'authentic.

${ }^{17}$ Gapps, 2009, p. 400.

18 Tony Horwitz, Confederates in the Attic: Dispatches from the Unfinished Civil War, New York, Pantheon Books, 1998, p. 7.

19 Jean-Marie Schaeffer, «De l'imagination à la fiction», Vox-Poetica, 2002, http://www.vox-poetica.org/t/articles/schaeffer.html (consultation le Io juillet 20I7). 
s'engage dans l'espace fictionnel des amorces qui lui permettent d'adopter l'attitude mentale du "comme si" ${ }^{20} \gg$.

I14 Deux autres procédés contribuent à instaurer une dialectique entre immersion et distanciation: l'art du double sens et le simulacre. Les reenactors multiplient les ruses de langage qui permettent à un même énoncé de signifier à la fois dans l'espace fictionnel et hors de lui, dans la réalité. Cette capacité à discourir simultanément sur des registres distincts permet de résoudre de façon ludique et humoristique les contradictions. Elle provoque chez les interlocuteurs des allers et retours entre incrédulité et crédulité. Dans le compte rendu de sa visite à la Plimoth Plantation et dans d'autres villages reconstitués, Richard Schechner est captivé par les interprètes qui «jouent, mais font semblant de ne pas jouer ${ }^{2 \mathrm{I}}{ }^{»}$, et mêlent étroitement leur vie vécue à leur vie jouée. Il y voit un véritable théâtre environnemental dans lequel les guides-acteurs et les visiteurs s'adonnent au plaisir des jeux de rôles et inventent ensemble des manières de résoudre les paradoxes. Dans les festivals d'histoire vivante dans lesquels les reenactors jouent dans un ordre chronologique de courtes saynètes représentatives des principales civilisations de la ligne du temps, les animateurs affectionnent ce double registre : ils racontent ce qui est en train d'être joué au présent, facilitant l'identification des performeurs et des spectateurs, mais interrompent régulièrement le fil de l'histoire par des jugements appréciatifs ou dépréciatifs sur la qualité du jeu ou sur les anachronismes heureux et malheureux.

Le deuxième procédé utilisé pour instaurer une dialectique entre ces régimes contradictoires est la production et la diffusion massives d'images opérant à la manière de simulacres. Les reenactors produisent une quantité phénoménale d'images photographiques, vidéographiques, filmiques qu'ils mettent en ligne sur les sites de leurs associations et sur les réseaux sociaux. Bien que la présence des appareils et des caméras lors des reenactments constitue un anachronisme criant, même les hardcore reenactors y recourent. Stephen Gapps explique que ces images contribuent à construire l'authenticité des reenactments ${ }^{22}$, selon la circularité propre au régime $\mathrm{du}$ simulacre. Celui-ci, Gilles Deleuze et Jean Baudrillard l'ont montrées , ne simule rien d'autre que lui-même et instaure un processus de répétitions, de réitérations interchangeables et équivalentes. Les images et les reenactments se reflètent selon un

\section{${ }^{20} \mathrm{Ibid}$.}

${ }^{21}$ Richard Schechner, 2008, p. 446.

${ }^{22}$ Gapps, 2009, p. 402.

${ }^{23}$ Gilles Deleuze, Logique du sens, Paris, Éditions de Minuit, 1969. Jean Baudrillard, Simulacres et simulation, Paris, Galilée, 198I. 
jeu de miroir dans lequel il n'y a ni modèle ni original. Les photographies de groupe, rituels obligés des reenactments, se présentent dès lors comme des tableaux vivants qui relancent cette circularité. Il est impossible d'établir ce qui des images ou des reenactments vient en premier. On retrouve le même usage de la photographie sur les sites Internet des musées d'histoire vivante. Ces images numériques aux couleurs rutilantes ne montrent généralement pas de touristes, mais seulement des protagonistes en costumes, comme s'il s'agissait de photographies qui avaient été prises à l'époque. Ces simulacres qui surjouent les anachronismes favorisent paradoxalement l'immersion et l'identification.

Le reenactment dans ses formes les plus élaborées combine donc «l'identification sympathique» ou empathique, indispensable à toute compréhension historique selon Robin George Collingwood, et l'effet de distanciation du théâtre épique de Bertolt Brecht, qui vise à rompre l'illusion et l'identification pour favoriser l'esprit critique ${ }^{24}$. C'est pourquoi il est erroné d'assimiler les reenactors et autres aficionados de l'histoire vivante à des nostalgiques qui cherchent à retourner dans le passé sans aucune distance critique.

L'importance des procédés d'immersion, d'identification et de simulation dans l'économie des reenactments explique la facilité avec laquelle les nouveaux médias s'en sont emparés, en particulier les émissions de téléréalité, les jeux vidéo et les univers virtuels. À la fin des années 1990, prolongeant la mode des docudrames et docufictions, les premières séries de téléréalité historique sont lancées. On invite des volontaires à revivre des événements qui ont marqué l'histoire ou à se projeter dans des périodes du passé. Parmi les grands succès, on compte en Angleterre The 1900 House (1999) et The 1940 House (200I), produites par Wall to Wall et diffusées sur Channel 4 et PBS. Dans la première, un couple et ses quatre enfants vivent durant trois mois dans un cadre simulant la fin de la période victorienne; dans la deuxième, une famille londonienne de classe moyenne survit dans des conditions similaires aux mois de raids aériens de l'aviation allemande durant la Seconde Guerre mondiale. The Ship (2002), diffusé sur BBC Two, propose une reconstitution du voyage de l'explorateur britannique James Cook, de Cairns sur la côte Est de l'Australie à Jakarta en Indonésie, sur son fameux trois-mâts, l'Endeavour. Sous la supervision de

${ }^{24}$ Robin George Collingwood, The Idea of History [1946], Oxford, Oxford University Press, 1993. Bertolt Brecht, « Description succincte d'une nouvelle technique d'art dramatique produisant un effet de distanciation », dans Écrits sur le théatre [1940], Paris, Gallimard, coll. «Bibliothèque de la Pléiade », 200o, p. 906. Sur le jeu de l'empathie et de la distanciation, voir également Vanessa Agnew, « History's Affective Turn: Historical Reenactment and its Work in the Present », Rethinking History, vol. II, $\mathrm{n}^{\mathrm{o}}$ 3, septembre 2007, p. 299-3I2. 
professionnels, cinquante-cinq hommes et femmes de nationalités différentes naviguèrent durant six semaines à bord d'une réplique du bateau, selon les techniques de l'époque. Au Québec, la chaîne Historia a inauguré en janvier 2017 sa première téléréalité, Le lot $d u$ diable, produite par Zone 3. Quatorze participants rejouent l'installation de colons sur des terres abandonnées par leurs prédécesseurs en AbitibiTémiscamingue, lors de la dépression des années 1930. Quelques mois plus tard, dans la lignée de The Ship, Radio-Canada télé lance La grande traversée (2017), une coproduction des Productions Rivard et Zone 3. Six hommes et quatre femmes y refont la traversée de l'Atlantique, de La Rochelle à Québec, à bord du trois-mâts l'Espérance, dans des conditions similaires à celles des premiers colons français venus s'établir en Nouvelle-France. Le ressort dramatique de ces téléséries repose sur la capacité des participants à s'immerger dans des situations difficiles et à dépasser leurs limites physiques et psychologiques, sur la réactivation d'épisodes fondateurs des identités nationales ou régionales, sur l'éveil des affects et des émotions à l'égard de l'histoire, sur des formes de socialité réarticulant l'individualisme et la communauté.

Les univers virtuels, jeux vidéo ou environnements multi-utilisateurs comme Second Life, entretiennent quant à eux le fantasme de rejouer la vie réelle, passée ou actuelle, d'en produire la simulation la plus parfaite possible. Brothers in Arms ou Call of Duty rejouent les batailles de Normandie après le débarquement des alliés durant la Seconde Guerre mondiale (voir la figure 2). Assassin's Creed Unity permet aux joueurs d'évoluer dans le Paris de la Révolution de ${ }^{7789}$. Les développeurs modélisent de façon toujours plus efficace les objets, les espaces construits et naturels, mais aussi les personnages, la manière dont ils se déplacent, interagissent, perçoivent, ressentent ${ }^{25}$. L'implication de plusieurs milliers de joueurs simultanément permet d'orchestrer des batailles au déploiement complexe et de retenir l'attention des participants. Ces progrès incessants favorisent l'immersion et l'identification. Même si les libertés prises à l'égard du passé sont grandes, et si l'issue des événements reste bien sûr incertaine, ces jeux vidéo abordent l'histoire en tant qu'expérience collective et immersive, comme les reenactments. Certains concepteurs recourent aux registres du documentaire et des archives afin d'amplifier les effets de vérité. Pour Brothers in

\footnotetext{
${ }_{25}$ Sur les liens entre les jeux vidéo et le reenactment, voir entre autres: Brian Rejack, « Toward a Virtual Reenactment of History: Video Games and the Recreation of the Past », Retbinking History, vol. II, $\mathrm{n}^{\mathrm{O}}$ 3, septembre 2007, p. 4II-425; Tero Pasanen, « Grinding for War: Authenticity and Experience in WWII MMOs », Ennen ja nyt, $\mathrm{n}^{\circ} \mathrm{I}$, II janvier 20I7, http://www.ennenjanyt.net/2017/or/grinding-for-war-authenticity-and-experience-in-wwiimmos/ (consultation le ro juillet 2017).
} 


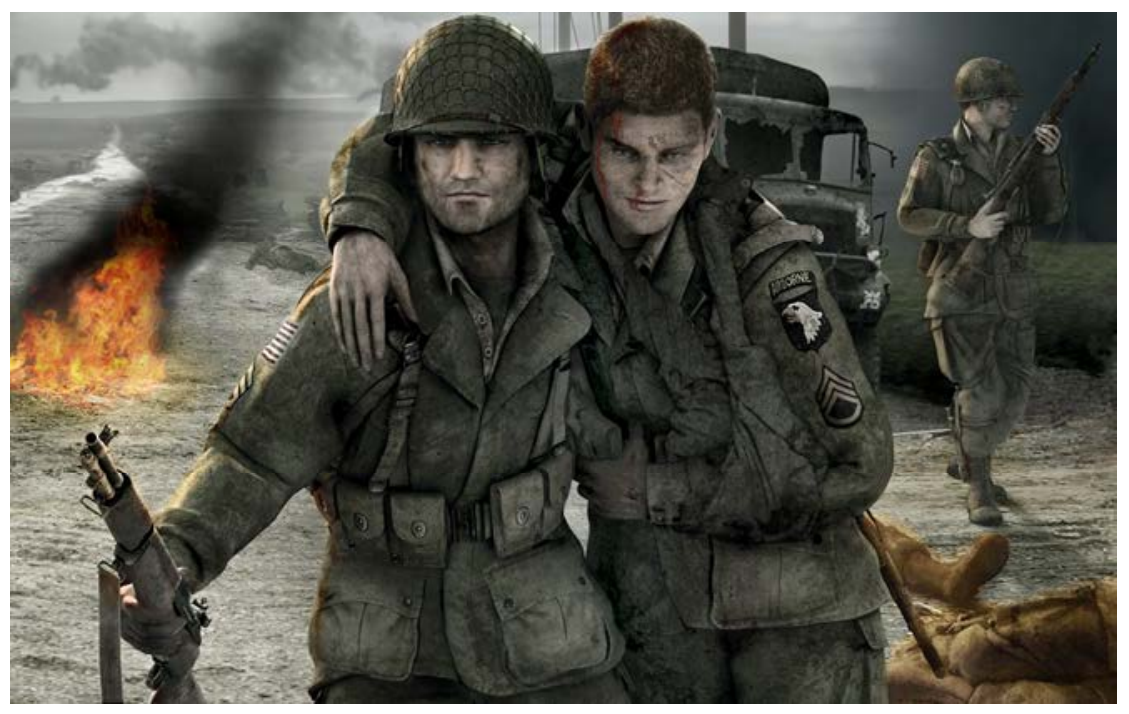

Fig. 2. Gearbox Software (développeur), Ubisoft (éditeur), Brothers in Arms, Hell's Highway, 2008, http://www.myplaystationwallpapers.net/I280xio24/brothers-in-arms-hells-highway03-artwork-wallpaper/.

Arms, l'équipe de Gearbox Software a intégré aux extras et au matériel promotionnel des entretiens, des photographies, des extraits de films d'archives, des captations de reconstitutions jouées qu'ils ont glanées sur le terrain en Normandie. Si ces ajouts confèrent au jeu une plus-value éducative et pédagogique, leur traitement tend à niveler les images modélisées et les documents d'archives, brouillant les registres du simulacre et de l'indiciel, soumettant le deuxième à la circularité du premier ${ }^{26}$.

Créé par le Linden Lab à San Francisco en 1999 et lancé en 2003, l'univers virtuel Second Life propose, comme son nom l'indique, une « deuxième vie » aux joueurs ${ }^{27}$. Ceux-ci personnalisent à volonté leurs avatars et mènent de multiples activités dans des espaces de vie qu'ils façonnent à l'aide d'une interface de design visuel. Si la plateforme exalte l'imagination et la créativité des utilisateurs (son slogan

\footnotetext{
${ }^{26}$ Rejack, 2007.
}

${ }^{27}$ Sur Second Life, voir entre autres : Tom Boellstorff, Coming of Age in Second Life: An Anthropologist Explores the Virtually Human, Princeton, Princeton University Press, 2008; Phylis Johnson, Second life, Media, and the Other Society, New York, Peter Lang, 2oro; Holly Willis, «The Unexamined Second Life Isn't Worth Living: Virtual Worlds and Interactive Art », Afterimage, vol. 35, $\mathrm{n}^{\circ}$ 2, septembre-octobre 2007, p. 13-16; Morgane Tual, « Absurde, créatif et débauché : dix ans après, "Second Life" est toujours vivant », Le Monde, 28 avril 2or6, http://www.lemonde.fr/pixels/article/2016/04/28/absurde-creatif-et-debauche-dix-ansapres-second-life-est-toujours-bien-vivant_4909910_4408996.html_(consultation le Io juillet 20I7). 
est « Your World, Your Imagination »), elle ressemble étrangement à notre monde, comme si elle en était la réplique virtuelle. On y trouve des reproductions de lieux réels, le Capitole ou le Mont-Saint-Michel. Les Slifers se fréquentent, s’invitent les uns chez les autres, organisent des soirées. Ils s'adonnent aux plaisirs de la consommation sur les avenues et dans les centres commerciaux. Les galeries, les musées, les centres d'art organisent des expositions et des soirées de performances; les salles de spectacles y présentent de grands concerts; etc. Une véritable économie s'y développe: on peut vendre, acheter ou louer des biens (immobiliers, culturels, de consommation courante, etc.) avec une monnaie virtuelle, le Linden dollar.

Les emboîtements avec les mondes matériels dans lesquels nous vivons sont nombreux et complexes. Le Linden dollar est convertible en dollar américain. Certains utilisateurs tirent leurs revenus du commerce de leurs créations ou des services qu'ils offrent dans l'univers virtuel. Les partis politiques se servent de Second Life comme d'une plateforme de communication. John Kerry et Barack Obama y ont mené campagne. Des conflits sociaux et politiques sont rejoués sur Second Life : lors de la campagne présidentielle française de 2007, une émeute contre le quartier général du Front national éclata; en 2009, des manifestants israéliens et palestiniens s'affrontèrent dans les rues; en 20II, Occupy Second Life est créé en soutien au mouvement Occupy Wall Street (voir la figure 3). Second Life reenacte le monde actuel et vice versa, selon une circularité propre au régime du simulacre. Dans le monde des nouveaux médias, le reenactment relève davantage de procédés de simulation, d'identification et d'immersion que de distanciation. Il est dès lors légitime de se demander si ces univers permettent de générer une réflexion critique, et comment. J'y reviendrai.

\section{«COMPORTEMENT RESTAURÉ »ET « TOURNANT AFFECTIF DE L'HISTOIRE "}

Bien qu'à peine esquissée, cette généalogie démontre la persistance d'un phénomène et la sophistication de ses procédés. Il est étonnant que les universitaires ne s'y soient intéressés qu'à la toute fin du $20^{\mathrm{e}}$ siècle. Deux facteurs expliquent cette indifférence selon Gapps ${ }^{28}$. D'une part, le reenactment a mauvaise presse, car il est souvent considéré comme l'expression d'une attitude nostalgique simpliste ou d'un sentiment nationaliste et conservateur. D'autre part, il est tombé dans un vide épistémologique, entre les disciplines du théâtre et de l'histoire.

${ }^{28}$ Stephen Gapps, « History and Performance: An Introduction », Performing the Past: A Cultural History of Historical Reenactments., 2002, p. I-49. 


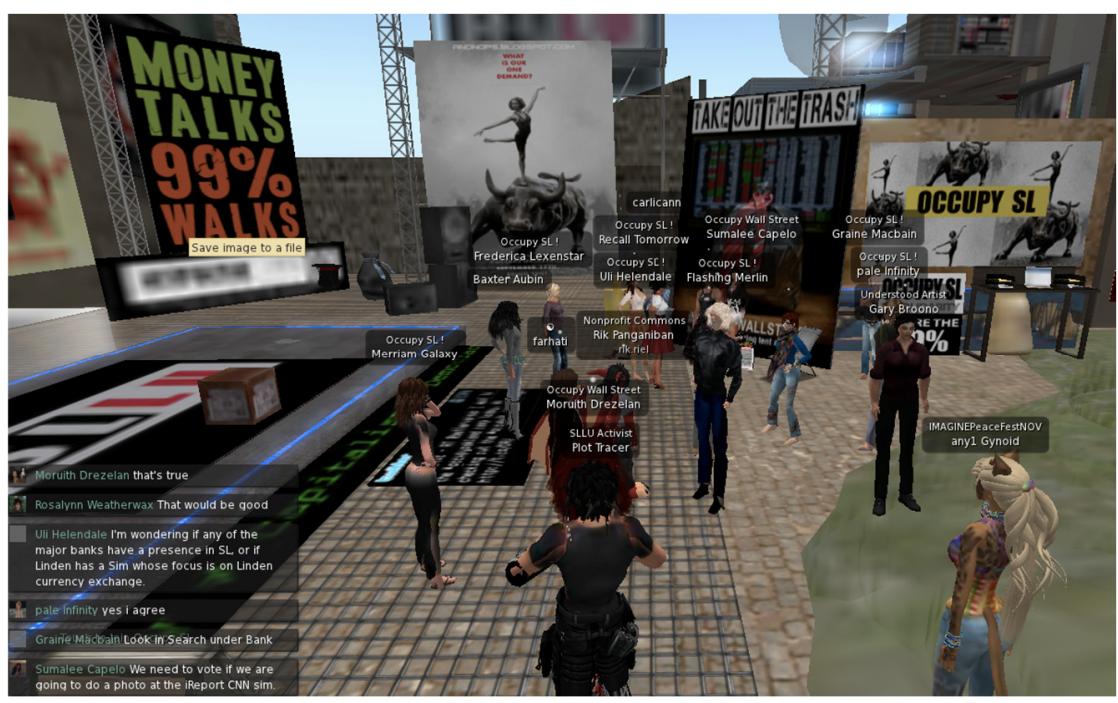

Fig. 3. Second Life, Occupy SL Planning Meeting, Flickr, mise en ligne par Rik Panganiban, https://www.flickr.com/photos/rikomatic/6262766266/in/pool-occupysl/, téléchargée le 20 octobre 2 OII.

Les premières analyses du reenactment sont issues des performance studies, champ d'études interdisciplinaires (même antidisciplinaires) qui se constitue en 1980 avec la fondation d'un premier département à la Tisch School of the Arts à l'Université de New York. Le metteur en scène américain Richard Schechner, qui réfléchissait alors aux liens entre le théâtre et le rituel, et l'anthropologue britannique Victor Turner, qui étudiait les rites de passage dans les cérémonials africains, en ont développé les idées fondatrices ${ }^{29}$. Ils inscrivent ce nouveau champ d'études dans la lignée du «tournant performatif » des sciences humaines et de la linguistique, notamment les travaux d'Erving Goffman et de John L. Austin ${ }^{30}$. Même si elle n'est pas nommée ainsi, la question du reenactment est d'emblée centrale, car elle est intriquée dans le concept même de performativité. Goffman situe le performatif entre les actes inscrits dans le réel et les actes fictionnels d'un comédien sur scène. L'individu en société possède un savoir social incorporé et inconscient similaire au savoir scénique de l'acteur; ses routines quotidiennes répètent des scenarii. À partir

29 Richard Schechner, Performance Studies: An Introduction, Londres, Routledge, 2002.

${ }^{30}$ John Langshaw Austin, Quand dire, c'est faire [1962], traduction de Gilles Lane, Éditions du Seuil, Paris, 1970. Erving Goffman, La mise en scène de la vie quotidienne [1959], traduction de Alain Accardo, Paris, Éditions de Minuit, 1973. 
de ces prémisses, Schechner a développé la notion de « comportement restauré » ou « comportement vécu deux fois ». «Tout comportement humain, écrit-il, est une recomposition de comportements précédents ${ }^{31} \gg$. Il est à la fois répétitif et réflexif parce que la répétition exige l'apprentissage social de gestes et de paroles à l'intérieur de cadres spécifiques. Les modalités de restauration du comportement sont toutefois multiples: conservation, manipulation, transformation, réinvention d'un passé historiquement avéré ou fictif, situé ou non localisable, etc.

Les générations suivantes de théoriciens des performance studies intègrent à cette réflexion les idées de Jacques Derrida sur le pouvoir subversif de la répétition des énoncés performatifs ${ }^{32}$. Si l'efficacité des actes de langage dépend de circonstances et de contextes appropriés et repose sur des conventions réglées par les institutions, Derrida montre que les procédés d'itération et de dérive permettent de déborder et de déjouer ces règles. À partir du double héritage de Schechner et de Derrida, deux avenues se sont ouvertes. La première considère le reenactment en tant qu'instrument d'agentivité au sens du terme anglais agency, qui désigne la capacité des sujets à agir sur leur réalité sociale; la deuxième consiste à le penser comme une arme contre l'autorité de l'archive.

Judith Butler tient une place centrale dans le développement du concept d'agentivité et de ses liens avec le reenactment. Notion complexe, souvent mal comprise, l'agentivité ne s'inscrit pas dans une téléologie de l'émancipation. Elle est paradoxalement intriquée au concept d'assujettissement selon lequel le pouvoir est à la fois oppresseur et créateur du sujet ${ }^{33}$. Dans ses réflexions sur le genre, Butler montre comment la répétition peut générer des formes d'agentivité. Les genres féminin et masculin ont une structure imitative. Leur répétition leur confère un statut d'origine et une légitimité en tant que norme : «[Le] genre est le mécanisme par lequel une norme de la différence sexuelle [...] est inculquée, incorporée [...], un code de pouvoir

${ }^{31}$ Richard Schechner, « La restauration du comportement », 2008, p. 398-399.

${ }^{32}$ Jacques Derrida, Marge de la philosophie, Paris, Éditions de Minuit, 1972.

33 Judith Butler, La vie psychique du pouvoir: l'assujettissement en théories [1997], traduction de Brice Matthieussent, Paris, Éditions Léo Scheer, 2002. Judith Bultler, Ces corps qui comptent. De la matérialité et des limites discursives du sexe [1993], traduction de Charlotte Nordmann, Paris, Amsterdam, 2009. «Agency: un concept opératoire pour les études de genre », dossier thématique, Rives méditerranéennes, $\mathrm{n}^{\circ} 4 \mathrm{I}, \quad 2 \mathrm{OI} 2$, https://rives.revues.org/4084 (consultation le ro juillet 2017). 
qui vise sans cesse à se reproduire et à s'instituer ${ }^{34} \gg$. Comment les corps peuvent-ils se défaire des normes de la différence sexuelle et devenir le lieu d'une agentivité ? Le queer est l'une des réponses possibles, selon Butler. Par le travestissement, il met en lumière la structure imitative des genres et, ce faisant, la mine. Il retourne l'insulte et l'outrage en se les appropriant et en les revendiquant comme identité propre. Il en fait l'espace même à partir duquel développer une revendication, une lutte, une «puissance d'agir transformatrice 35 ». L'agentivité consiste alors à « trouver la liberté dans une marge de manœuvre à déployer face aux prescriptions ${ }^{36} \gg$ afin de resignifier et de réélaborer les normes.

C'est selon un processus similaire que le reenactment, puissant moyen de confirmation des valeurs dominantes et de manipulation idéologique, peut générer des formes d'agentivité. À travers la répétition, il transmet des connaissances, des savoirs, des conceptions du monde; il confirme des ordres symboliques et sociaux, des valeurs philosophiques, politiques, esthétiques, morales, etc. Il génère des formes d'identification et des sentiments d'appartenance à un groupe. À travers lui, le sujet incorpore et institue des règles et des normes. Mais il a toujours la possibilité de performer autrement, de suspendre ces logiques de confirmation et d'adhésion, de déconstruire les normes, d'en jouer, de les déjouer, de les transformer, d'y résister, de les resignifier, de les réélaborer.

Cette intrication de l'agentivité à l'assujettissement et à la normalisation permet de réévaluer les univers virtuels. Dans Coming of Age in Second Life, Tom Boellstorff observe des formes d'agentivité virtuelle dans Second Life ${ }^{37}$. Comme de nombreux jeux vidéo, la plateforme renforce les normes de genre. Les utilisateurs doivent choisir un sexe féminin ou masculin pour leurs avatars et les configurer avec des outils fortement stéréotypés. Pourtant, Boellstorff constate de fréquentes réinventions des identités. Certains choisissent d'incarner un avatar de l'autre sexe sans révéler leur genre aux autres usagers. Des couples hétérosexuels se transforment en couples homosexuels. Les possibilités de créer des avatars de sexes différents et de passer de l'un à l'autre grâce à la fonction « alt » engendrent des formes de socialité

\footnotetext{
34 Monique Haicault, « Autour d'agency. Un nouveau paradigme pour les recherches de genre », Rives méditerranéennes, $\mathrm{n}^{\circ}$ 4I, 2012, p. 18, http://rives.revues.org/4IO5 (consultation le ro juillet 2017).

35 Butler, 2009.

${ }^{36}$ Haicault, 2012, p. I8.

37 Boellstorff, 2008.
} 
transgenre. Ou encore, l'apparence de certains avatars ne permet de les rattacher à aucun genre.

La deuxième avenue ouverte par les relectures de Schechner à travers Derrida est une réévaluation de l'autorité de l'archive par le biais du reenactment. Rebecca Schneider, André Lepecki et Diana Taylor comptent parmi les voix importantes. Ils cherchent à dépasser la posture de Peggy Phelan qui rattache la performance à une ontologie de la perte et exclut de son champ la saisie documentaire et les phénomènes de réitération ${ }^{38}$. Intriquant la notion de «comportement restauré » et l'idée de potentiel subversif de la réitération, Schneider soutient que la performance en tant qu' « acte de rester et de réapparaître » perdure dans le temps à travers sa répétition 39. Le reenactment serait dès lors une « forme performative d'archive » apparentée au symptôme et à la contre-mémoire. Il permettrait de produire des contre-histoires et de donner une parole aux groupes minoritaires. Il réhabiliterait les savoirs des corps que les traditions occidentales ont écartés. Lepecki, quant à lui, avance la notion de «volonté d'archiver» à propos des reenactments d'œuvres performatives et chorégraphiques, et dissocie la danse et la performance de l'idée de perte ontologique ${ }^{40}$. Au lieu de fixer les œuvres dans une singularité originale à la manière des entreprises de documentation, les reenactments les ouvrent, les délient, activent les champs de possibilités ou de virtualités qu'elles portent en elles. Ils opèrent également un recouvrement de l'archive et du corps. Le corps devient une archive vivante qui, par sa mobilité, sa précarité, son indétermination, résiste à la domiciliation et à l'autorité de l'auteur sur son œuvre. Le corps-archive est dès lors un espace de réinvention et de réécriture des œuvres. Enfin, Taylor rattache le reenactment au répertoire, un ensemble de gestes transmis par le corps à travers des pratiques vivantes, selon un processus pleinement créatif de répétitions et de différences ${ }^{41}$. Alors que l'archive stabilise et fixe, le répertoire conserve tout en transformant. Il a une portée épistémologique, car il valorise les savoirs incorporés, les diffuse de manière transnationale et transhistorique. Il est facteur d'agentivité parce que les modifications et les mutations que les acteurs opèrent leur permettent de se réinventer en fonction des changements qui surviennent dans leur société et dans leur environnement. Si ces auteurs reconnaissent pleinement la fonction

${ }^{8}$ Peggy Phelan, Unmarked: the Politics of Performance, Londres, Routledge, 1993.

39 Rebecca Schneider, Performing Remains: Art and War in Times of Theatrical Reenactment, Abingdon, Routledge, 2011.

$4^{\circ}$ Lepecki, 20I5.

${ }^{41}$ Diana Taylor, The Archive and the Repertoire: Performing Cultural Memory in the Americas, Durham, Duke University Press, 2003. 
mémorielle du reenactment et revendiquent même une dialectique de l'archive et du vivant, du direct et du médiatisé pour le penser, c'est dans la perspective d'analyser sa capacité à subvertir la logique de l'archive et l'économie des médias. Pour eux, l'agentivité advient à travers la performativité du reenactment. Ou elle est menacée par le régime de l'archive et des médias, ou elle cherche à le miner.

Dans les années 200o, une nouvelle génération d'historiens ouverts aux études culturelles s'intéresse à son tour au reenactment. Leurs préoccupations croisent à plusieurs égards celles des théoriciens des performance studies. Vanessa Agnew constate un « tournant affectif de l'histoire ${ }^{42}$ » qui marque la « fin de la négligence de l'histoire savante à l'égard des affects $43 \gg$. Avant d'être un ensemble de connaissances, l'histoire serait de l'ordre des sensations. «Forme d'histoire affective », le reenactment saisit les émotions liées à une situation passée et en produit de nouvelles. Il permet que les sensibilités historiques se révèlent et s'expriment. Comment le passé nous affecte-t-il ? De quelle manière ces sentiments éprouvés et incorporés, sensations de fierté, d'appartenance, de rejet, de honte, d'injustice, etc., influencent-ils nos actions et notre compréhension de l'histoire et de l'actualité ?

Gapps insiste sur la portée politique du reenactment. Il pousse les groupes sociaux et culturels à renégocier les récits historiques et contribue ainsi à résoudre les conflits et les tensions d'aujourd'hui ${ }^{44}$. Plus inclusif que les formes traditionnelles d'historiographie, le reenactment redonne une parole aux sans-voix, aux marginalisés et aux vaincus de l'histoire. Il leur offre l'occasion de revendiquer leurs droits. En Australie, au Canada et aux États-Unis, il provoque des débats sur la place des aborigènes dans les représentations de l'histoire coloniale ou sur l'exclusion des mémoires et des corps des femmes dans les pratiques d'histoire vivante.

Pour être constructives, ces discussions ne peuvent se contenter d'être inclusives. Elles doivent contribuer à penser la complexité des renégociations des récits historiques : «There is [...] a fine line between participation that suggests complicity with the historical project and participation that involves working the politics of visibility45 », écrit Gapps. Plusieurs tactiques sont préconisées. L'inversion des rôles consiste, par exemple, à faire jouer le capitaine Cook par un aborigène, ou des confédérés et des soldats de l'armée britannique par des Afro-Américains. Ces

\footnotetext{
${ }^{42}$ Agnew, 2007.

${ }^{4}$ Ibid., p. 300.

44 Gapps, 2009. Gapps, 2002.

45 Gapps, 2009, p. 405.
} 
renversements rendent visible la blanchitude (whiteness) comme hégémonie sociale, politique et culturelle. À la manière des cultural white studies, ils en révèlent les processus de construction institutionnelle et médiatique. Une deuxième tactique, l'uchronie, permet d'envisager le passé comme un ensemble de « possibilités confisquées », au sens de ce qui n'a pu advenir, et de le réinventer selon des «interprétations plus fructueuses ${ }^{46} \gg$. Par exemple, les rencontres interculturelles des siècles passés peuvent y prendre d'autres formes que le colonialisme. Ces reconstitutions imaginaires interrogent les verrouillages culturels et sociaux, et les intérêts qu'ils desservent.

Des débats similaires se tiennent dans les musées d'histoire vivante. Aux ÉtatsUnis, dans les années 1980, la direction du Colonial Williamsburg souhaita restituer une visibilité aux Afro-Américains qui constituaient au $18^{\mathrm{e}}$ siècle 52 pour cent de la population. Ils engagèrent des membres des communautés noires pour incarner des esclaves, une situation qui reconduisait les préjugés raciaux, faisait naitre des sentiments de honte et d'humiliation chez les performeurs, et souleva des protestations de la part des communautés noires et blanches. Rex M. Ellis, acteur afro-américain et professeur d'études théâtrales intéressé aux théâtres militants et engagés, développa The Other Half Tour (la visite de l'autre moitié), une visite guidée de deux heures montrant la vie à Williamsburg du point de vue des esclaves ${ }^{47}$. Il incita les interprètes des esclaves à sortir des conventions de leur rôle et à s'exprimer sur l'histoire des Afro-Américains, à en raconter les récits, les luttes pour les droits civiques et la dignité. Autrement dit, ils adoptèrent une double posture d'énonciation : l'esclave du $18 \mathrm{e}$ siècle (identification) et l'Afro-Américain du $20^{\mathrm{e}}$ siècle qui prend en charge son propre récit historique et élabore une critique de la représentation qu'il accepte d'incarner (distanciation). Ces expériences menées par les musées d'histoire vivante pour inclure les voix afro-américaines et autochtones sont déterminantes dans le développement d'une muséologie des droits de la personne aux États-Unis ${ }^{48}$.

Cette nouvelle génération d'historiens intrique beaucoup plus aisément que les théoriciens des performance studies le performatif et le médiatisé dans leurs

${ }^{46}$ Vanessa Agnew, «Introduction: What Is Reenactment? », Criticism, vol. 46, n 3 , été 2004 , p. 328.

47 Marcus Wood, « Historic Williamsburg: Theatre, Memory, and Colonial Slavery », dans Julia Swindells et David Francis Taylor (dir.), The Oxford Handbook of The Georgian Theatre I737-I832, Oxford, Oxford University Press, 20I4, p. 706-725 (en particulier la section « Rex M. Ellis at Williamsburg », p. 7II-715). Anna Logan Lawson, "The Other Half": Making AfricanAmerican History at Colonial Williamsburg, thèse de doctorat, University of Virginia, 1995.

${ }^{48}$ Notamment le projet du Smithsonian National Museum of African American History and Culture inauguré en 2016 (Rex M. Ellis en est alors le directeur adjoint). 
réflexions sur l'agentivité du reenactment. Celle-ci ne saurait advenir sans une prise en considération des chaînes intermédiales des représentations historiques. Les travaux d'Agnew sur les téléréalités historiques des années 2000, notamment les adaptations allemandes des séries britanniques House (Abenteuer 1900 et Abenteuer 1927), sont à cet égard éclairants ${ }^{49}$. Ces émissions cherchent davantage à « surmonter le présent ${ }^{50}$ 》 que le passé. Selon les procédés de l'anachronisme, le passé y est prétexte à penser le présent. Sous le couvert d'un retour au début du $20^{\mathrm{e}}$ siècle, Abentener Igoo et Abenteuer 1927 répondent aux clivages issus de la réunification de l'Allemagne. Pour que des formes de distanciation et d'agentivité émergent de ces téléréalités, il est essentiel qu'elles donnent à penser les chaînes intermédiales dans lesquelles elles s'inscrivent. D'autant plus que l'économie de la production télévisuelle dont elles relèvent est basée sur les adaptations, les prequels, les sequels et autres reprises qui reconduisent inlassablement les mêmes récits, représentations et interprétations. Lorsqu'elles ne sont pas inscrites dans ces généalogies, les téléréalités historiques tombent dans un relativisme sans portée critique, selon Agnew.

\section{LES REENACTMENTS ARTISTIQUES OU LE RÉPERTOIRE EN RÉGIME INTERMÉDIAL}

Les pratiques artistiques semblent particulièrement propices à explorer le potentiel critique du reenactment en régime intermédial. Depuis le début des années 2000 , le reenactment a envahi les mondes de l'art sous des formes qui conjuguent le performatif et le médiatisé, et dans une perspective revendiquée de rejouer l'histoire pour interroger ses processus de fabrication, ses généalogies et le pouvoir de ses représentations. Les artistes voient dans le reenactment la possibilité de relancer le travail sur l'archive qu'ils ont mené au cours des années 1990 et 2000. Aux expositions marquantes qui ont été organisées sur la culture archivistique, Deep Storage: Collecting, Storing, and Archiving in Art, Interarchiv ou Archive Fever's, etc., répondent plusieurs expositions d'envergure sur le reenactment: Life Once

\footnotetext{
49 Agnew, 2004, p. 327-339.

so Agnew joue ici sur l'expression «coming to terms with the past» ou Vergangenheitsbewältigung, le fait de surmonter le passé.

${ }^{51}$ Deep Storage. Collecting, Storing, and Archiving in Art, Haus der Kunst, Munich, 3 août-I2 octobre 1997, P.S. I Contemporary Art Center, New York, 5 juillet-30 août 1998, Henry Art Gallery, Seattle, 5 novembre-3I janvier 1999. Interarchiv, Kunstraum, Universitât Lüneburg, Lüneburg (1997-2002). Archive Fever: Uses of the Document in Contemporary Art, International Centre of Photography, New York, I8 janvier-4 mai 2008.
} 
More. Forms of Reenactment in Contemporary Art, History Will Repeat Itself. Strategies of Re-enactment in Contemporary (Media) Art and Performance ou Re:Akt! Reconstruction, Re-enactment, Re-reportings', etc.

Il semble toutefois imprudent de soutenir que les usages artistiques du reenactment sont dotés d'une portée critique dont les reenactments non artistiques seraient dépourvus. Cette assertion mise trop souvent de l'avant accorde à l'artiste une intelligence dont le reenactor serait dépourvu. La culture du reenactment est riche et complexe, nous l'avons vu. Depuis le début du $20^{\mathrm{e}}$ siècle, ses acteurs se sont dotés d'une littérature réflexive qui en élabore les diverses conceptions, approches et méthodologies ${ }^{33}$. Les organisateurs de reenactments ainsi que leurs participants sont de plus en plus sollicités pour leurs connaissances et leurs savoir-faire par l'industrie cinématographique et télévisuelle, par les organismes patrimoniaux et par les artistes. Il m'apparait plus juste d'envisager les reenactments artistiques à la jonction de deux pratiques culturelles. Ils conjuguent les savoirs des reenactors et la maitrise des chaînes intermédiales de l'art contemporain, selon une acception du reenactment que j’appelle « le répertoire en régime intermédial » et que j'expliciterai à partir de The Battle of Orgreave (200I), de l'artiste britannique Jeremy Deller.

Cette œuvre ambitieuse et intermédiale se déploie sous quatre formes : un reenactment co-organisé avec Howard Gils; un film coréalisé avec Mike Figgis, diffusé sur Channel 4 et dans les salles de musées; une installation acquise en 2005 par le musée londonien Tate Modern (voir les figures 4 et 5); et un livre édité par Artangel. Reenactment artistique parmi les plus commentés, The Battle of Orgreave recrée l'affrontement à Orgreave, une cokerie du nord-est de l'Angleterre dans le South Yorkshire, le 18 juin 1984 , entre les mineurs en grève et les forces policières sous le gouvernement de Margaret Thatcher. Plusieurs milliers de mineurs reculèrent devant

${ }^{52}$ Life Once More. Forms of Reenactment in Contemporary Art, Witte de With, Rotterdam, 27 janvier-27 mars 2005. History Will Repeat Itself. Strategies of Re-enactment in Contemporary (Media) Art and Performance, KW Institute for Contemporary Art, Berlin, I8 novembre 2007-13 janvier 2008. Re:Akt! Reconstruction, Re-enactment, Re-reporting, National Museum of Contemporary Art, Bucharest, 22 janvier-I3 mars 2009, ŠKUC Gallery, Llubljana, 25 mars-17 avril 2009, Maribor Art Gallery, Maribor, 21 octobre-19 novembre 2010, http://www.reakt.org/ (consultation le 8 octobre 20I7).

53 Par exemple, à la suite du succès que connut en 1905 le pageant de Sherborne (Angleterre) organisé par Louis N. Parker, un livre fut publié pour en garder la mémoire et expliciter l'approche préconisée par Parker : Cecil P. Goodden, The Story of The Sherborne Pageant: Produced in the Old Castle Ruins at Sherborne in June, 19o5, Sherborne, F. Bennett, The Parade, 1905. 
une force policière imposante, lourdement équipée et entraînée au combat, qui déploya des moyens et une violence disproportionnés. Le bilan fut très lourd pour les mineurs : des blessés, certains graves, des arrestations et des condamnations. Orgreave marqua l'affaiblissement des syndicats face au gouvernement Thatcher. L'événement se transforma en conflit politique et idéologique polarisé par les médias. Deller souhaitait réviser les récits médiatiques de cette lutte et donner une parole aux mineurs dans cette relecture.

Les analyses du reenactment de Deller insistent sur le fait que l'artiste impliqua des mineurs et quelques policiers qui avaient vécu l'événement dix-sept ans plus tôt, et tint la recréation à proximité du site original, favorisant ainsi un dialogue entre les partis et avec l'ensemble de la communauté. Si ces aspects sont essentiels au potentiel critique et cathartique de l'entreprise, il faut toutefois rappeler que Deller, soutenu financièrement par la fondation anglaise Artangel, engagea la compagnie EventPlan, spécialisée dans la recréation d'événements historiques pour la télévision et le cinéma. Son fondateur et directeur Howard Giles, reenactor réputé, prit en charge l'organisation du reenactment. Il recruta et entraîna plus de Iooo participants, parmi lesquels 280 mineurs et policiers impliqués dans l'événement de 1984 et 800 《extras » choisis parmi plus de quarante sociétés de reenactment de batailles historiques à travers toute l'Angleterre. En faisant appel à ce milieu, Deller s'assurait de compétences indispensables à son projet, mais il cherchait également à faire entrer la bataille d'Orgreave dans la culture du reenactment et dans le répertoire des batailles dignes d'être reconstituées : «I wanted the re-enactment of The Battle of Orgreave to become part of the lineage of decisive battles in English History ${ }^{54} \gg$, explique-t-il. Corollairement, l'expérience fut marquante pour les communautés de reenactors. La possibilité de rencontrer les acteurs de l'histoire à rejouer était non seulement exceptionnelle, mais ouvrait des pans immenses de questionnements et de réflexions sur leur propre pratique. Ils étaient confrontés aux points de vue contradictoires des vainqueurs et des vaincus sur un événement historique récent dont les récits et les représentations médiatiques étaient éminemment polémiques. Avec The Battle of Orgreave, Deller inscrit la négociation au cour même de la notion de répertoire.

54 Jeremy Deller, « Foreword », The English Civil War Part II. Personal Accounts of the 1984-85 Miners'Strike, Londres, Artangel, 200I, p. 7. 

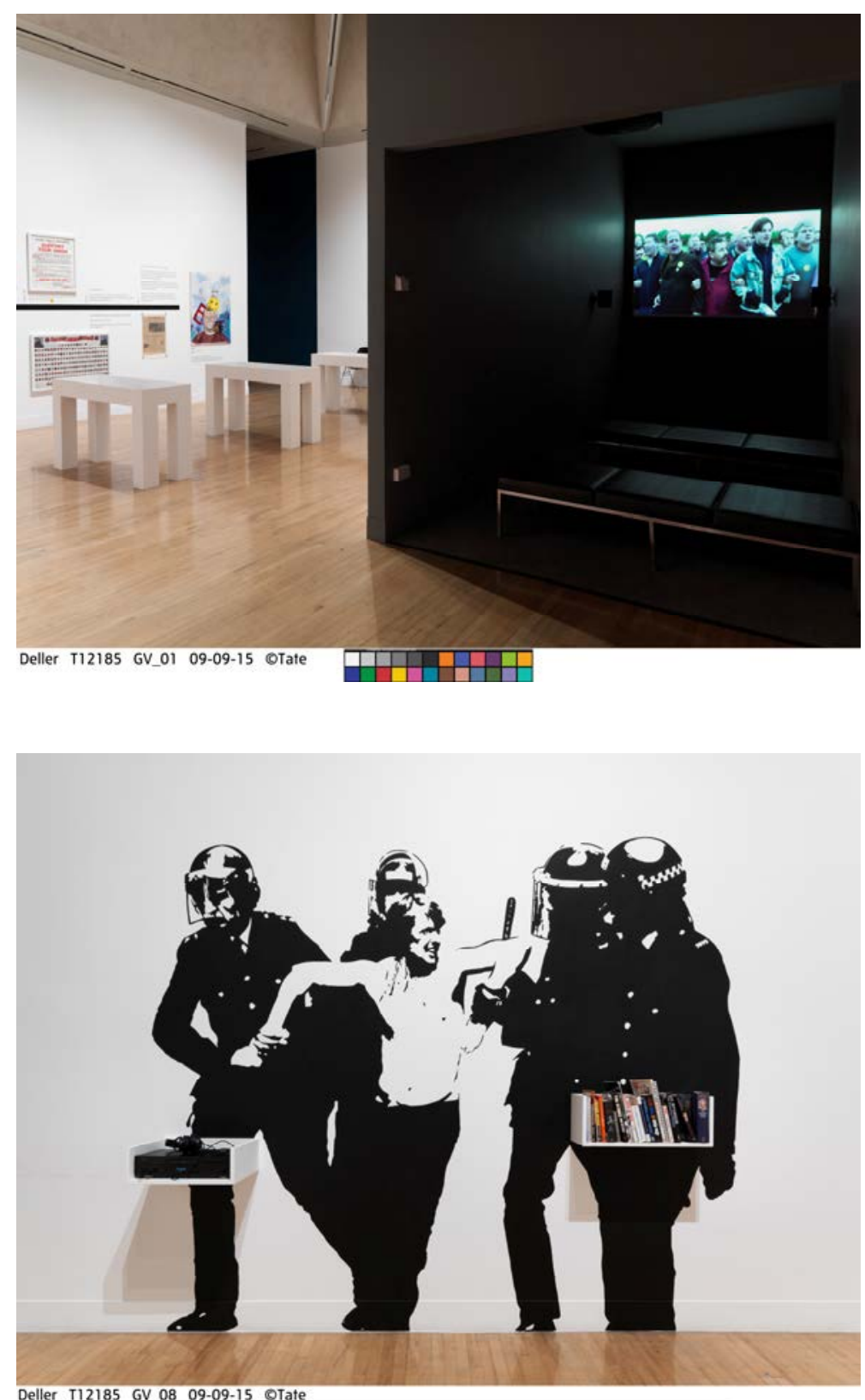

Fig. 4-5. Jeremy Deller, The Battle of Orgreave Archive (An Injury to One is an Injury to All), installation, Tate Modern, Londres, 20or (detail). Avec l'aimable autorisation de Jeremy Deller et de la Tate Modern.@ Jeremy Deller. 
Le répertoire est une notion partagée par tous les arts vivants, même s'il est difficile d'en arrêter une définition'ss. À l'inverse d'une collection ou d'un patrimoine que l'on conserve, et contrairement au canon qui est constitué d'un ensemble d'œuvres sacralisées connues et admirées, le répertoire est rejoué, recyclé, actualisé. Il est évolutif, expansif, dynamique ${ }^{56}$. Comme Taylor, les anthropologues et les sociologues empruntent la notion de répertoire pour analyser les rôles joués par les individus dans la société ou pour étudier les modalités des actions collectives protestataires. Dans son anthropologie du citadin, Ulf Hannerz différencie l'inventaire des rôles offerts aux individus par leur communauté des répertoires qu'ils y jouent ${ }^{57}$. Cette distinction vise à montrer que les citadins ne sont pas condamnés à choisir parmi des rôles disponibles, mais peuvent en créer de nouveaux. Ann Swidler envisage la culture comme un répertoire actif de comportements, de croyances et d'actions que les membres d'un groupe sélectionnent et utilisent pour élaborer leurs stratégies et prendre leurs décisions ${ }^{5}$. Sorte de boîte à outils, le répertoire lui permet de développer un paradigme de la culture tourné vers l'action. Charles Tillys9 avance quant à lui la notion de répertoire d'actions contestataires pour désigner les moyens que les acteurs mobilisent dans les conflits sociaux. À la fois déterminées et indéterminées, les formes protestataires sont en nombre limité, mais sans cesse réinventées et remotivées. Dans les sciences sociales, le répertoire permet donc d'insister sur l'idée de choix délibéré parmi un ensemble de possibilités et d'invention de nouvelles modalités.

Certains jugent toutefois cette acception limitée, car « le choix [est toujours] pris dans un nœud d'interactions avec les choix d'autres acteurs, [...] les autorités notamment $t^{60} \gg$. Ils préfèrent parler en termes de négociation. Dans le domaine du

s5 Frédéric Maurin montre la complexité et les fluctuations de la notion de répertoire dans les arts vivants: Frédéric Maurin, «L'impermanence est-elle soluble dans le répertoire ?», L'annuaire théatral: revue québécoise d'études théatrales, ${ }^{\mathrm{os}} 53-54,2013, \mathrm{p} .75-90$.

56 Valentina Litvan et Marta López Izquierdo, «Répertoire(s). Mode d'emploi », Pandora, $\mathrm{n}^{\mathrm{0}}$ 7, 2007, p. 9-17.

57 Ulf Hannerz, Explorer la ville. Éléments d'anthropologie urbaine [1980], traduction de Isaac Joseph, Paris, Éditions de Minuit, 1983.

${ }^{58}$ Anne Swidler, «Culture in Action: Symbols and Strategies », American Sociological Review, vol. 5I, avril 1986, p. 273-286.

59 Charles Tilly, Regimes and Repertoire, Chicago, University of Chicago Press, 2006. «Ouvrir le "répertoire d'action". Entretien avec Charles Tilly», Vacarme, no $3 \mathrm{I}$, printemps 2005, p. 2I-22.

6o Michel Offerlé, «Retour critique sur les répertoires de l'action collective ( $18^{\mathrm{e}}-$ $2 \mathrm{I}^{\mathrm{e}}$ siècles) », politix, vol. 8I, $\mathrm{n}^{\mathrm{O}} \mathrm{I}, 2008$, p. I8I-202. 
jazz, les sociologues Robert Faulkner et Howard Becker avancent l'expression « répertoire en action » pour désigner les processus de négociation et « d'ajustement mutuel » aux autres et à l'environnement ${ }^{6 \mathrm{I}}$. « [II] s'agit d'un procédé compliqué qui nécessite la participation de plusieurs personnes [...] et qui implique des discussions et des négociations, parfois implicites ${ }^{62} \gg$ : propositions, contre-propositions, questions, mises au point, etc.

De nombreuses séquences du film de Figgis et Deller témoignent de ces processus de négociation : les réunions des anciens mineurs et des «extras »; les répétitions; les entretiens avec les différents protagonistes, ceux de 1984 (mineurs, policiers, syndicalistes, groupes de soutien, etc.) et ceux de $200 \mathrm{I}$ (mineurs rejouant leur propre rôle ou celui de policiers selon le renversement préconisé par Gapps, enfants de mineurs, extras, organisateurs, etc.). The Battle of Orgreave inscrit la négociation au cœur du répertoire sans pour autant qu'elle n'aboutisse à un consensus. Il s'agit de montrer les ressorts des disputes et des dissensus, les affects qui les sous-tendent : on passe de la colère au rire, de la rancour à la réconciliation, de la résignation à la révolte, on assiste à la transmission transgénérationnelle de ces émotions, etc.

The Battle of Orgreave est également un objet intermédial complexe. Outre les multiples médiums artistiques qu'il convoque, il intrique l'expérience vécue, les archives et les médias. Les processus de négociation du répertoire y opèrent entre les acteurs, mais aussi à travers les chaînes intermédiales de représentation. Le matériel rassemblé et les discours sont hétérogènes : des témoignages des protagonistes de 1984 et de 20oI; des photographies de 1984 en noir et blanc; des captations en couleurs du reenactment de 20or, d'autres en noir et blanc; des déclarations d'époque de Margaret Thatcher à la télévision et à la radio; une transcription des excuses publiques de la BBC en 1991 reconnaissant la partialité de sa couverture médiatique des affrontements de 1984; des analyses stratégiques de l'affrontement; des souvenirs et des reliques; etc. Selon Ralph Rugoff, à travers cette profusion, Deller souhaite « to openly acknowledge that any history is inevitably impure, highly mediated, and in need of

${ }^{6}$ Robert R. Faulkner et Howard S. Becker, Qu'est-ce qu'on joue maintenant? Le répertoire de jazz en action [2009], traduction de Bruno Gendre, Paris, La Découverte, 2oII.

62 Camille Azaïs, Talia Bachir-Loopuyt et Pierre Saint-Germier, «Du jazz aux mouvements sociaux : le répertoire en action. Entretien avec Howard Becker », traduction de Camille Azaïs, Tracés. Revue de sciences humaines, no I8, 20I0, http://traces.revues.org/4625 (consultation le is juillet 2017). 
being re-written ${ }^{63} \gg$. Cette page de l'histoire sociale de l'Angleterre n'en finit d'ailleurs pas d'être révisée. En 2012, la BBC diffusa le documentaire The Inside Out, qui corrobore les doutes quant à la collusion du gouvernement Thatcher et de la police du South Yorkshire. Après avoir promis en juin 20I5 de lancer l'enquête publique réclamée par les mineurs et leurs familles depuis trente ans, Theresa May fit toutefois volte-face quelques mois plus tard ${ }^{64}$.

The Battle of Orgreave fait entrer un affrontement issu d'un mouvement de contestation sociale dans le répertoire des grandes batailles britanniques à rejouer; elle l'inscrit dans des chaînes intermédiales infinies qui la débordent et auxquelles elle contribue, au croisement de perspectives multiples et contradictoires. Le performatif, l'archive et les médias y sont étroitement intriqués, et cet enchevêtrement instaure une dialectique de l'immersion et de la distanciation qui permet des formes d'agentivité. Des acteurs qui ont des intérêts et des cultures différentes sont amenés à négocier les représentations historiques aussi bien vivantes que médiatiques. Cette logique de transmission est celle du répertoire en régime intermédial, c'est-à-dire un processus de négociation des représentations historiques vivantes et médiatiques qui ne vise pas le consensus, mais cherche au contraire à déverrouiller les interprétations hétérogènes et contradictoires, les intérêts et les affects dont elles sont porteuses. Il ne fait pas travailler le vivant contre l'archive, ni l'archive contre le vivant, mais les enchevêtre pour que le sens œuvre contre lui-même, selon la belle formule de Jacques Rancière6s.

C'est cette acception du reenactment que les collaborateurs de ce numéro d'Intermédialités ont été invités à explorer et à discuter, non pas en tant que concept, mais comme agencement de questions. Comment comprendre et analyser les relations entre le reenactment, les médias, les technologies, les archives et les institutions? De quelle manière aborder le corps qui refait, traversé d'affects et de mémoires kinesthésiques, aux prises avec ces appareils ? Selon quelles modalités les jeux de l'immersion et de la distanciation, de l'assujettissement et de l'agentivité opèrent-ils dans les reenactments en régime intermédial ? L'intrication du vivant et du médiatisé dans les pratiques actuelles de reenactment permet-elle une redistribution

${ }^{63}$ Ralph Rugoff(dir.), Jeremy Deller. Joy in People, catalogue d'exposition, Londres, Hayward Gallery, 2012, p. 17.

64 David Conn, «The Scandal of Orgreave», The Guardian, I8 mai 2017, https://www.theguardian.com/politics/2017/may/r8/scandal-of-orgreave-miners-strikehillsborough-theresa-may (consultation le ro juillet 2017).

6s Rancière définit le consensus comme « l'accord entre sens et sens ». Jacques Rancière, Le spectateur émancipé, Paris, La fabrique éditions, 2008, p. 75. 
des responsabilités et des pouvoirs dans la transmission de la culture ? À partir d'un large éventail de pratiques de reenactment, et selon un champ sémantique très étendu de la notion, les auteurs élaborent des échafaudages théoriques et méthodologiques permettant d'appréhender ces enjeux et, surtout, d'en saisir la complexité. Certaines contributions examinent, dans une perspective théorique, les liens du reenactment avec le simulacre et l'inscrivent dans un «renversement du platonisme» (Vaillancourt), ou encore proposent des outils analytiques pour appréhender les réitérations médiales des industries cinématographiques et télévisuelles, et leurs prolongements dans les réseaux de communication participatifs (Boni). À la croisée de ces interrogations, le dossier d'artiste présente une œuvre qui s'élabore sur les lieux des conflits internationaux, mais toujours en retard sur les journalistes, afin de pouvoir reenacter leurs procédés de médiatisation des événements (Licha). D’autres contributions appréhendent le reenactment à travers le prisme des affects. Comment les émotions qui bouleversent la puissance d'agir des corps se transmettent-elles à travers un film d'animation, ou au fil de chaînes intermédiales (Fevry et Wouters; Chare), et inversement du document d'archives au théâtre (Wind) ? Comment les langages oraux et corporels traversés d'affects et le document se croisent-ils dans une pratique d'entretien d'artiste en art de la performance (Barbut) ? Le souvenir de la réception d'une pièce chorégraphique et la manière dont elle nous touche en tant que spectateur peuvent-ils être remis en jeu et faire œuvre de danse (Le Boulba) ? Plusieurs explorent les diverses manières dont les pratiques culturelles de tradition orale s'inscrivent dans les environnements médiatiques: la diffusion des patrimoines immatériels sur Internet aux prises avec les exigences politiques des États et de l'UNESCO, ou avec les contraintes des algorithmes et des intérêts financiers privés de YouTube (Pietrobruno); l'évolution récente du fado au regard de ses traditions et des exigences de l'industrie du disque (Patrix); les outils technologiques d'apprentissage du tango qui articulent l'improvisation aux « diffractions médiales » (De Luca). Enfin, d'autres auteurs analysent les passages entre le vivant et le médiatisé, entre les archives et les mémoires orales et corporelles. Des corpus de gestes standardisés et rationalisés à des fins d'efficacité et de rentabilité technologiques et économiques peuvent-ils faire répertoire de création (Prunet) ? Comment le cinéma, la télévision expérimentale et les arts visuels s'emparent-ils de l'histoire vivante dans ses formes commerciales et folkloriques (Fourgeaud) ? De quelles manières et à quelles fins le reenactment peut-il reconstituer, réactiver ou remettre en acte une collection (Fraser et Dubé-Moreau) ?

L'auteur remercie Steve Giasson pour son aide précieuse à la recherche. 


\section{Le reenactment ou le répertoire en régime intermédial}

AnNe BÉnichou, Université de Montréal

\section{NOTE BIOGRAPHIQUE}

ANNE BÉNICHOU est professeure d'histoire et de théorie de l'art à l'École des arts visuels et médiatiques ainsi qu'au programme d'études supérieures en muséologie de l'Université du Québec à Montréal. Ses recherches portent sur les archives, les formes mémorielles et les récits historiques issus des pratiques artistiques contemporaines et des institutions chargées de les préserver et de les diffuser. Elle poursuit actuellement des travaux sur les nouvelles formes de documentation et de transmission des œuvres éphémères et performatives. Elle a publié Muntadas. Between the Frames: the Forum (Musée d'art contemporain de Barcelone, 20II) et Un imaginaire institutionnel. Musées, collections et archives d'artistes (Paris, L'Harmattan, coll. « Esthétiques », 2013). Elle a dirigé les ouvrages collectifs Ouvrir le document. Enjeux et pratiques de la documentation dans les arts visuels contemporains et Recréer/Scripter. Mémoires et transmissions des auvres performatives et chorégraphiques contemporaines (Dijon, Les presses du réel, 20Io et 20I5). Ses écrits récents ont paru, entre autres, dans les revues Performance Research, Ligeia et Thema, ainsi que dans le magazine Ciel Variable, pour lequel elle a dirigé le numéro « Documents de performance ». 